\title{
Mortality Audit of Octogenarians With Acute Cholecystitis
}

\author{
Arkadiusz Peter Wysocki, FRACS, Jennifer Allen, BSc(Hons), Therese Rey-Conde, MPH, \\ John B. North, FRACS \\ Department of Surgery, Logan Hospital, Meadowbrook, Queensland, Australia (Dr. Wysocki). \\ Queensland Audit of Surgical Mortality, Royal Australasian College of Surgeons, East Brisbane, Queensland, Australia \\ (Drs. Allen, Rey-Conde, and North).
}

\begin{abstract}
Background and Objectives: The mortality rate of patients with acute cholecystitis is low with either medical or surgical management. It is unclear how surgeons decide which patients will not undergo cholecystectomy. We postulated those who died following medical management would have a greater burden of comorbidities than those who died following cholecystectomy.

Methods: Adults who died under the care of a surgeon with a diagnosis of acute cholecystitis were identified from the Australian and New Zealand Audit of Surgical Mortality database.

Results: We identified 86 eligible patients, and two-thirds of them were managed medically. Cholecystectomy patients were younger (78 years vs 86 years, $P=.028$ ) and had a lower American Society of Anesthesiologists class $(3$ vs $4, P=$ .005). Both groups had a similar number of comorbidities $(P=.588)$. Length of stay for the surgical group was 11 days longer than that of the medical group (14 days vs 3 days, $P<.001$ ). The frequency of hospital systems issues was the same in both groups.

Conclusions: Patients with acute cholecystitis who died with medical management were older with a higher American Society of Anesthesiologists class than those who died following cholecystectomy. Research is required into the circumstances at time of admission for acute cholecystitis.
\end{abstract}

Key Words: Mortality, Audit, Acute cholecystitis, Laparoscopy, Cholecystectomy.

Citation Peter Wysocki A, Allen J, Rey-Conde T, North JB. Mortality audit of octogenarians with acute cholecystitis. CRSLS e2014.00098. DOI 10.4293/CRSLS.2014.00098.

Copyright (C) 2014 SLS This is an open-access article distributed under the terms of the Creative Commons Attribution-Noncommercial-ShareAlike 3.0 Unported license, which permits unrestricted noncommercial use, distribution, and reproduction in any medium, provided the original author and source are credited.

Contributions: A.P.W. and J.B.N. developed the idea; J.A. and T.R.C. collected the data; A.P.W., J.A., and T.R.C. interpreted the data; and all authors contributed to the writing and revisions of the manuscript.

Address correspondence to: A. Peter Wysocki, FRACS, Logan Hospital, Corner Armstrong and Loganlea Roads, Meadowbrook, Queensland 4131, Australia. Telephone: +61-7-3299-8899, Fax: +61-7-3299-8535, E-mail: arek_p@ecn.net.au

\section{INTRODUCTION}

With the adoption of acute surgical care units, suitable patients with acute cholecystitis often undergo early laparoscopic cholecystectomy. Patients deemed unsuitable for an operation (by surgeon or anesthetist) are managed conservatively. What is not clear from published series, is under what circumstances patients were admitted and on what grounds some were considered to be unfit for general anesthesia: for example, not all patients with American Society of Anesthesiologists (ASA) class 4 or higher undergo medical management alone. ${ }^{1}$ For example, in 1 acute surgical care unit with protocol-driven patient care, only $80 \%$ of patients with acute cholecystitis underwent surgical procedures as opposed to $96 \%$ of those with right iliac fossa pain (with suspected acute appendicitis). ${ }^{2}$ A selection bias exists.

Current literature lacks consensus on the optimal approach to the unwell and/or unfit patient with severe acute cholecystitis, and management decisions are largely driven by personal preference (ie, medical management, 
percutaneous drainage, cholecystectomy). We postulate that surgeons opt for medical management in preference to surgical care for patients who are deemed too frail. However, Revenig et al ${ }^{3}$ have recently suggested the decision whether or not a patient is fit for an operation may be based on an erroneous assessment of the patient's physiological state. We designed this study to test our hypothesis that patients who died with conservatively managed acute cholecystitis were older and had a greater burden of comorbidities than did those who died following surgical management.

\section{MATERIALS AND METHODS}

Data was prospectively collected through the ANZASM (Australian and New Zealand Audit of Surgical Mortality) database over the 4 years from January 1, 2009, through December 31, 2012. This study covers the continent of Australia (currently 99\% of all public teaching hospitals and $73 \%$ of private hospitals take part). Deaths are reported to ANZASM by the hospital if the patient was an inpatient at the time of death and under the care of a surgeon, whether or not a surgical procedure was performed. Patients who were readmitted under a surgeon and died within 30 days of a surgical procedure are also included. Patients admitted for palliative treatment are excluded from the ANZASM database. ANZASM is a protected quality assurance activity in Australia under Part VC of the Health Insurance Act 1973 (established August 2011). Ethical approval was obtained from the Royal Australasian College of Surgeons Ethics Committee.

The functioning, governance, and objectives of ANZASM have been outlined elsewhere. ${ }^{4}$ The treating surgeon provides the clinical data to ANZASM using a standard surgical case form. The deidentified surgical case form is sent for first-line assessment to a surgeon from a different hospital (same specialty). Based on clinical judgment (without a standardized pro forma), the assessor surgeon determines whether there were any adverse events and whether deficiencies in standard surgical care arose (eg, communication issues, fluid balance issues, delays). The case may then be closed or proceed to a non-deidentified second-line assessment where a different assessor surgeon has access to the medical progress notes for that admission. As the surgical case form data is provided by treating surgeons, responses to questions are open to bias and not all questions may have been answered. The determinations of the assessor surgeons represent their own clinical opinions rather than that of ANZASM.
Data were included if the patient was 17 years or older and died in an Australian hospital with a final diagnosis of acute cholecystitis whether or not any surgery had been performed. The diagnosis was made by the treating team using clinical, laboratory, imaging results and, where applicable, operative findings. Patients managed medically generally receive intravenous antibiotics, fluids, thromboembolism prophylaxis, and sometimes cholecystostomy. Those managed operatively receive similar medical care together with cholecystectomy (laparoscopic or open). Patients who fail medical management may subsequently undergo cholecystectomy. Baseline patient population data were not available for public or private patients who were treated for acute cholecystitis (medically or surgically) and were discharged from hospital alive.

All data were extracted from the ANZASM database using IBM SPSS Statistics (version 19, IBM Corporation, Armonk, New York). Chart review was not possible. A nonparametric test was used to test the significance of a difference between groups using Microsoft Excel (Redmond, Washington). Differences in frequency counts between groups (binomial outcome) were tested using a Z-test. Continuous variables are presented as medians with interquartile ranges (IQR). Categorical variables are presented as frequencies with the percentages. Significance values were based on 2-tailed tests, with $P<.05$ considered statistically significant.

\section{RESULTS}

A total of 111 patients died with a primary diagnosis of acute cholecystitis. Five were managed by percutaneous cholecystostomy; 10 patients experienced diagnosis or treatment delays; and another 10 underwent cholecystectomy following failed medical management, that is, after 3 days of hospitalization (median day 7 [IQR 5, 16]). These 25 patients were excluded in order to have 2 comparable groups of patients that differ only by treatment strategy (ie, medical vs cholecystectomy) without crossover or confounders.

The remaining 86 patients belonged in 2 groups: 33 who underwent cholecystectomy and 53 who were treated medically without interventional radiology as demonstrated in Table 1. There were fewer women in the surgical group ( $45.5 \%$ vs $54.7 \%$, respectively) but this difference was not statistically significant $(P=.403)$. The operated group were considerably younger (median 78 years vs 86 years, respectively, $P=.028$ ). The surgical group had a longer length of stay (median 14 days vs 3 
Table 1.

Comparison of Patients Who Underwent Cholecystectomy and Nonoperative Management

\begin{tabular}{llll}
\hline Characteristics & Medical management $(\mathrm{n}=53)$ & Cholecystectomy $(\mathrm{n}=33)$ & $P$ value \\
\hline Female & $29(54.7)$ & $15(45.5)$ & $78(72,84)$ \\
Age & $86(77,91)$ & $14(5,29)$ & .028 \\
Length of stay & $3(2,8)$ & $3(2,4)$ & $<.001$ \\
Number of comorbidities & $4(2,4)$ & $3(3,4)$ & .588 \\
ASA class & $4(3,4)$ & $14(46.7)$ & .005 \\
Unplanned ICU admission $^{*}$ & $2(4)$ & $2(6.7)$ & .001 \\
Fluid balance issues $^{*}$ & $7(14.0)$ & $2(9.1)$ & .315 \\
Communication issues $^{\dagger}$ & $1(2.9)$ & $12(36.4)$ & .002 \\
Different management & $15(9.4)$ & & \\
\hline
\end{tabular}

Abbreviation: ASA, American Society of Anesthesiologists; ICU, intensive care unit.

Data are presented as $n$ (\%) or median (interquartile range).

*Data missing for 6 patients.

${ }^{\dagger}$ Data missing for 30 patients.

days, $P<.001)$ than did the medical group. The cholecystectomy group had fewer comorbidities than the medical group did, but the difference was not statistically significant (median 3 vs 4 , respectively, $P=$.588). The ASA class was lower in the cholecystectomy group (median 3 vs 4 , respectively, $P=.005$ ). Of the 9 patients with ASA class 5, 7 were in the medical group (18.4\% vs 6.3\%, $P=.148)$. A greater proportion of operated patients had an unplanned admission to the intensive care unit (46.7\% vs 4.0\%, $P<.001$ ). During the 2011 financial year, there were 7354 emergency admissions to Australian public hospitals due to cholelithiasis. ${ }^{5}$ Extrapolating from this, over the 4-year study period, 29416 patients were admitted acutely with cholelithiasis. Three-quarters of acute gallstone admissions are due to acute cholecystitis. ${ }^{6}$ The 111 patients identified in this study (prior to exclusions) yield a crude mortality for acute cholecystitis of $0.50 \%$.

In the medically treated group, the provisional clinical diagnosis was acute cholecystitis in 49 patients (92.5\%) and acute cholecystitis with perforation in 4 (7.5\%). None of the patients underwent an autopsy. In the surgical group, the diagnosis was acute cholecystitis in 50 patients (86.2\%), gangrenous cholecystitis in $6(10.3 \%)$, and empyema in $2(3.4 \%)$.

One-half of the patients (45.5\%) underwent cholecystectomy on the day of admission (median 1 day [IQR 0, 2]). Twenty-six patients underwent a laparoscopic cholecystectomy, but this was converted to open in $10(38.5 \%$ conversion rate). Seven underwent open cholecystectomy, but the reasons for not commencing laparoscopically were not provided. The following reasons for conversion from laparoscopy to open were recorded: postoperative adhesions, unexpected presence of cirrhosis, gangrenous cholecystitis, hemorrhage. Twenty-one patients experienced a definable postoperative complication (63.6\%), and 12 had no complications other than death. Complications recorded were 7 cardiorespiratory and 6 intra-abdominal, but complications data was missing for 8 patients.

Fluid balance issues were identified by the treating surgeon in $11.3 \%$ of patients overall, and this was the same across the 2 groups ( $P=.315$ ). Communication deficiencies were identified in $5.4 \%$ of all patients, and this was the same across the 2 groups $(P=.537)$. The surgeon responsible for the care of $36.4 \%$ of surgical patients felt that in retrospect they would have managed the patient differently as opposed to $9.4 \%$ of patients managed without surgery $(P=.002)$. Data for how the care would have differed is not available.

The length of stay for the nonoperative group was 3 days (IQR 2, 8), whereas for the cholecystectomy group, it was 14 days (IQR 5, 29) with this difference being highly statistically significant $(P<.001)$. The reported causes of death were in the following categories: ongoing sepsis in 29.1\% (25 patients), multiple organ failure in $25.6 \%$ (22 patients), cardiac in $22.1 \%$ (19 patients), respiratory in $9.3 \%$ (8 patients), and other 
in $14.0 \%$ (12 patients). There was no statistical difference in frequencies in the nonoperative and cholecystectomy groups (data not shown). However, patients who died following cholecystectomy due to sepsis or multiple organ failure had a dramatically longer length of stay than did those patients who were managed nonoperatively $(P<.05$ for each). Those who died from a cardiac, respiratory or other event (eg, cerebrovascular event, liver failure) had the same length of stay irrespective of treatment group as illustrated in Table 2 .

\section{DISCUSSION}

ANZASM is a protected quality assurance activity in Australia under Part VC of the Health Insurance Act 1973 (published August 2011) designed to improve patient outcomes. Our review of this national surgical mortality database revealed medically treated patients who died with acute cholecystitis were of an older age with a higher ASA class than were those who died following cholecystectomy. The number of comorbidities was the same in the 2 groups. The frequency of hospital systems issues (eg, communication issues, fluid balance issues) was the same in both groups.

Patients who were managed with cholecystectomy had a length of stay that was 11 days longer than that of the nonintervention group. Although the causes of death were the same in both groups, those who died from sepsis or multiple organ failure following cholecystectomy had a dramatically longer length of stay than did those who died following medical management. In keeping with this finding, the medically managed group was much less likely to be treated in the intensive care unit, reflecting a less aggressive treatment strategy in this group. These results suggest patients managed nonoperatively had clinically more severe comorbidities; were more physiologically compromised on presentation; or had a ceiling of care established on admission by the patient, family, and hospital staff due to these factors.
One-half of the patients who underwent cholecystectomy did so on the day of admission. Furthermore, three-quarters of the entire cholecystectomy cohort (prior to excluding patients who underwent cholecystectomy after day 3 of hospitalization or who experienced management delays) underwent cholecystectomy within the first 3 days of hospitalization, that is, in most patients, cholecystectomy was not a rescue procedure for failed medical management (37 of 50; data not shown). The complication rate of $63.6 \%$ in the cholecystectomy group seems much higher than the $35.1 \%$ overall rate for ANZASM data (unpublished data; 11101 deaths across all surgical specialties with median ASA class 4; IQR 3, 4). The almost 40\% conversion rate from laparoscopic to open cholecystectomy is double the already high $20 \%$ rate reported in a recent Cochrane review, ${ }^{7}$ suggesting this elderly cohort had a more hostile abdomen. The ANZASM database is unable to determine whether this relates to the duration of symptoms prior to admission, frequency of prior upper abdominal surgery, obesity, and/or prior acute cholecystitis managed medically. Delays in the complete ANZASM database are most frequently attributed to diagnostic support services with misinterpretation of results, incorrect tests being performed, and results not seen. ${ }^{8}$ Possible clinical reasons for the delay in diagnosis in the 10 excluded patients could include the following: acute coronary syndrome mimicking acute biliary pathology, for example, with increased troponin concentration'; lack of right upper quadrant tenderness in one-quarter of elderly patients with acute cholecystitis ${ }^{10}$; or normal white blood cell count in one-third of elderly patients with acute cholecystitis. ${ }^{10}$

The observation that complications in the elderly population are difficult to predict has led to the recent use of the concept of frailty to identify patients at risk. ${ }^{3}$ We did not use a frailty index in our study, but we used the number

Table 2.

Causes of Death and Length of Stay by Management Type

\begin{tabular}{llll}
\hline Causes of death & Medical management $(\mathrm{n}=53)$ & Cholecystectomy $(\mathrm{n}=33)$ & $P$ value \\
\hline Sepsis $(\mathrm{n}=25)$ & $3(1,4)$ & $29(16,47)$ & $16(11,22)$ \\
Multiple organ failure $(\mathrm{n}=22)$ & $6(4,9)$ & $3(2,4)$ & .001 \\
Cardiac $(\mathrm{n}=19)$ & $2(1,6)$ & $12(6,25)$ & .553 \\
Respiratory $(\mathrm{n}=8)$ & $13(9,16)$ & $12(9,31)$ & .539 \\
Other $(\mathrm{n}=10)$ & $8(2,9)$ & & .257 \\
\hline
\end{tabular}

Data are presented as length of stay in days (interquartile range). 
of comorbidities as a surrogate. The Hopkins Frailty Score-which takes into account unintentional weight loss ( $4.5 \mathrm{~kg}$ in last year), weakness, exhaustion, low physical activity, and slowed walking-is predictive of postoperative complications, length of stay, and discharge to a nursing facility. ${ }^{11}$ A recent proof-of-concept study demonstrated that unintentional weight loss was the most crucial frailty factor; frailty status was not predictive of severe (grade III or greater) complications; and preoperative anemia may also be a frailty marker. ${ }^{3}$ The overall mortality rate in that study was low (1.6\%), but none of the patients assessed as not frail died. ${ }^{3}$

The number of comorbidities was the same in both groups, but the ASA class was significantly lower in the surgical group. Although the ASA classification is known to suffer from interobserver variability, ${ }^{12}$ this finding suggests the latter group may have been more unwell as a result of their chronic comorbidities (or acute presentation) given that the number of comorbidities is a poor predictor of in-hospital mortality. ${ }^{13}$ The surgical group had a longer length of stay and a higher frequency of intensive care unit admission in keeping with this more aggressive treatment strategy (ie, surgical vs medical care). In addition, one-half of the surgical group underwent laparotomy (17 of 33), which in the setting of sepsis and significant comorbidities would result in admission to the intensive care unit as surgery is known to aggravate existing organ failure. ${ }^{14}$

Although early cholecystectomy for acute cholecystitis is generally accepted, ${ }^{7}$ recent research questions conventional wisdom when it comes to the elderly or medically unfit patient. ${ }^{15-18}$ First, percutaneous cholecystostomy may not be lifesaving. ${ }^{16}$ Second, delaying cholecystectomy in high-risk patients beyond the day of admission serves only to increase operative difficulty and prolong overall length of stay without reducing 30-day morbidity or mortality. ${ }^{15}$ Third, medical management of acute cholecystitis is associated with a higher 30-day mortality rate than that of cholecystectomy. ${ }^{17}$ And lastly, operating after 3 days of symptoms is just as safe as operating earlier. ${ }^{18}$

Although our study has the following limitations, we do not consider these prohibitive in attempting to address the question of how surgeons decide between operative and nonoperative management of acute cholecystitis: severity of premorbid comorbidities as well as the acute physiological dysfunction in both groups is unknown and may not be comparable. Additional limitations include the following: the use of an audit database, possible reporting bias, denominator variation due to not all questions having been answered, and including only in-hospital deaths while under the care of a surgeon. Even though the pathology in the intervention group has been confirmed, it is possible the provisional diagnosis in some of the patients in the medical group was not correct and another condition was misdiagnosed as acute cholecystitis. Not all comorbidities carry the same medical risks and although we can count the number of comorbidities per patient, the ANZASM database does not record the severity of comorbidities, which may differ between the 2 groups studied. The current study's greatest strength is that it includes all adults who died with a primary diagnosis of acute cholecystitis in Australia over a 4-year period. Data is systematically collected by surgeons using a standard self-reporting tool.

Findings from the Australian surgical mortality database suggest that that surgeons place emphasis on patient age and severity of pre-existing comorbidities when deciding between medical or surgical therapy for the management of acute cholecystitis. Further research is required into the circumstances at time of admission (eg, to the acute surgical care unit) for patients diagnosed with acute cholecystitis in order to understand the decision-making process.

\section{References:}

1. Nikfarjam M, Yeo D, Perini M, et al. Outcomes of cholecystectomy for treatment of acute cholecystitis in octogenarians. ANZ J Surg. 2013 Aug 1 [Epub ahead of print]. doi: 10.111/ ans. 12313 .

2. Cox MR, Cook L, Dobson J, Lambrakis P, Ganesh S, Cregan P. Acute Surgical Unit: a new model of care. ANZ J Surg. 2010; 80(6):419-424.

3. Revenig LM, Canter DJ, Taylor MD, et al. Too frail for surgery? Initial results of a large multidisciplinary prospective study examining preoperative variables predictive of poor surgical outcomes. J Am Coll Surg. 2013;217(4):665-670.e1.

4. Raju RS, Guy GS, Majid AJ, Babidge W, Maddern GJ. The Australian and New Zealand Audit of Surgical Mortality-Birth, Deaths, and Carriage. Ann Surg. 2014 Mar 18 [Epub ahead of print].

5. Australian Institute of Health and Welfare. Australian Hospital Statistics: 2011-12. Canberra: Australian Institute of Health and Welfare; 2013.

6. Cheruvu CV, Eyre-Brook IA. Consequences of prolonged wait before gallbladder surgery. Ann R Coll Surg Engl. 2002; 84(1):20-22. 
7. Gurusamy KS, Davidson C, Gluud C, Davidson BR. Early versus delayed laparoscopic cholecystectomy for people with acute cholecystitis. Cochrane Database Syst Rev. 2013;6: CD005440.

8. North JB, Blackford FJ, Wall D, et al. Analysis of the causes and effects of delay before diagnosis using surgical mortality data. Br J Surg. 2013;100(3):419-425.

9. Demarchi MS, Regusci L, Fasolini F. Electrocardiographic changes and false-positive troponin I in a patient with acute cholecystitis. Case Rep Gastroenterol. 2012;6(2): $410-414$.

10. Morrow DJ, Thompson J, Wilson SE. Acute cholecystitis in the elderly: a surgical emergency. Arch Surg. 1978;113(10): 1149-1152.

11. Makary MA, Segev DL, Pronovost PJ, et al. Frailty as a predictor of surgical outcomes in older patients. J Am Coll Surg. 2010;210(6):901-908.

12. Haynes SR, Lawler PG. An assessment of the consistency of ASA physical status classification allocation. Anaesthesia. 1995; 50(3):195-199.

13. Escobar GJ, Greene JD, Scheirer P, Gardner MN, Draper D, Kipnis P. Risk-adjusting hospital inpatient mortality using automated inpatient, outpatient, and laboratory databases. Med Care. 2008;46(3):232-239.

14. Saadia R, Schein M. Multiple organ failure. How valid is the "two hit" model? J Accid Emerg Med. 1999;16(3):163-166; discussion 166-167.

15. Brooks KR, Scarborough JE, Vaslef SN, Shapiro ML. No need to wait: an analysis of the timing of cholecystectomy during admission for acute cholecystitis using the American College of Surgeons National Surgical Quality Improvement Program database. J Trauma Acute Care Surg. 2013;74(1):167-173; 173-174.

16. Gurusamy KS, Rossi M, Davidson BR. Percutaneous cholecystostomy for high-risk surgical patients with acute calculous cholecystitis. Cochrane Database Syst Rev. 2013;8: CD007088.

17. Riall TS, Zhang D, Townsend CM Jr, Kuo YF, Goodwin JS. Failure to perform cholecystectomy for acute cholecystitis in elderly patients is associated with increased morbidity, mortality, and cost. J Am Coll Surg. 2010;210(5):668-677, 677-679.

18. Degrate L, Ciravegna AL, Luperto M, et al. Acute cholecystitis: the golden 72 -h period is not a strict limit to perform early cholecystectomy. Results from 316 consecutive patients. Langenbecks Arch Surg. 2013;398(8):1129-1136. 